\title{
Modelo predictivo inteligente para la generación de energía eléctrica en un sistema fotovoltaico
}

\section{Intelligent forecast model for electrical power generation in a photovoltaic system}

\author{
ARELLANO-VALMAÑA, Fernando*†, RUZ-HERNANDEZ, Jose Antonio y PITALÚA-DÍAZ, Nun
}

Universidad Autónoma del Carmen - Departamento de Ingeniería y Tecnología

ID $1^{\text {er }}$ Autor: Fernando, Arellano-Valmaña / ORC ID: 0000-0003-4799-6354, Researcher ID Thomson: F-8025-2019, CVU CONACYT ID: 669418

ID $1^{\mathrm{er}}$ Coautor: José Antonio, Ruz-Hernández / ORC ID: 0000-0001-8332-4980, CVU CONACYT ID: 216374, SNI CONACYT: 41306

ID $2^{\text {do }}$ Coautor: Nun, Pitalúa-Díaz / ORC ID: 0000-0002-8671-1422, Researcher ID Thomson: G-1230-2019, CVU CONACYT ID: 100050

\section{Resumen}

La energía solar es hoy en día la fuente renovable de mayor recurso mundialmente donde su obtención, a diferencia de muchas otras utilizadas actualmente, no supone ningún daño para el medio ambiente. Administrar satisfactoriamente la demanda de electricidad por sistemas fotovoltaicos requiere una planeación cuidadosa, sin embargo, definir el comportamiento solar e incluso predecir la radiación solar incidente es un proceso completamente estocástico debido a la influencia de variables meteorológicas. Para superar este problema, este trabajo monitorea diversas variables atmosféricas a través de una estación ubicada en la Universidad de Sonora en Hermosillo, México; y registra mediciones en tiempo real cada 5 minutos a lo largo de 6 meses. A partir de los datos obtenidos se presenta el desarrollo de un método computacional aplicando un sistema de inferencia difusa basado en redes neuronales (ANFIS) como técnica inteligente para predecir la potencia eléctrica de un sistema fotovoltaico instalado en el mismo sitio. Es de vital importancia entender la influencia de las variables meteorológicas en el consumo de energía, donde un mejor entendimiento de ellas puede contribuir en una estrategia de mayor utilidad para alcanzar la eficiencia energética para México.

Sistema fotovoltaico, variables meteorológicas, Sistema de inferencia difusa basado en redes neuronales (ANFIS)

\begin{abstract}
Solar energy is currently the most resourceful renewable source worldwide in which its obtainment, unlike many others currently used, does not mean any harm to the environment. To manage satisfactorily the electricity demand of photovoltaic systems a careful planning is required, nonetheless, to set successfully the solar behavior and even more to predict the amount of incident solar radiation is a completely stochastic process due the influence of meteorological variables. To overcome this problem, this study monitors diverse atmospheric variables by using a meteorological station located in the University of Sonora in Hermosillo city, Mexico; and registers real-time measure values every 5 minutes for 6 months. From the data obtained a computational model applying an Adaptive Neuro Fuzzy Inference System (ANFIS) as intelligent technique is presented in order to forecast the electrical power from a photovoltaic system installed in the same site. It is vital to understand the influence of meteorological variables on energy consumption in which a better understanding of it can contribute to a more useful strategy in meeting energy efficiency goal for Mexico.
\end{abstract}

Photovoltaic system, meteorological variables, Adaptive Neuro Fuzzy Inference System (ANFIS)

Citación: ARELLANO-VALMAÑA, Fernando, RUZ-HERNANDEZ, Jose Antonio y PITALÚA-DÍAZ, Nun. Modelo predictivo inteligente para la generación de energía eléctrica en un sistema fotovoltaico. Revista de Ingeniería Eléctrica. 2019. 3-10: $22-29$

$\dagger$ Investigador contribuyendo como primer Autor.

*Correspondencia al Autor correo electrónico: (060038@mail.unacar.mx) 


\section{Introducción}

La creciente demografía global implica un aumento en la demanda energética, siendo insostenible si sólo se obtiene a través de recursos no renovables, llevando a un encarecimiento de los servicios. La energía solar es actualmente la fuente que mayor recurso tiene mundialmente (Dudley, 2018). El proceso de producción no supone daños al medio y la cantidad de recursos aprovechables supera a cualquier otra energía considerada. (Elshurafa et al., 2018; Philibert, 2011; Rasero, 2011; WenTao et al., 2017).

La forma en que obtienen, transforman y distribuyen la radiación solar son los criterios más importantes por los cuales se caracterizan las tecnologías solares. De igual manera, se consideran las características de los sistemas fotovoltaicos para almacenar la energía generada (Järvelä et al., 2017). Una vez generada la potencia, gracias al uso de paneles solares, se suministra para alimentar la red eléctrica (Awan et al., 2018; Perea-Moreno et al., 2018; Zahedi, 2006).

México es un país con alta incidencia de radiación solar, con gran extensión territorial (dos de los aspectos más importantes en cuanto a generación fotovoltaica) y con estudios en energía solar; sin embargo, actualmente el nivel de investigación se encuentra por debajo de ciertas potencias tales como Alemania, Italia, España, Estados Unidos y China (Awan et al., 2018; Kazem et al., 2016; Wen-Tao et al., 2017; Zahedi, 2006).

Sin lugar a dudas, México tiene mucho potencial en cuanto a energía solar se refiere; si se comparara con Europa, el único país con una notoria incidencia de radiación es España alcanzando valores máximos entre 4.8 y 5.4 $\mathrm{kWh} / \mathrm{m}^{2}$ (ADRASE, 2018). Aun así, México excede a España alcanzando un promedio entre 5.6 y $6.2 \mathrm{kWh} / \mathrm{m}^{2}$ en la región noroeste, superando incluso a China quien en su mayoría contempla valores de $4.6 \mathrm{kWh} / \mathrm{m}^{2}$ (RuzHernandez et al., 2019).

Sin embargo, el obtener exitosamente el comportamiento solar e incluso predecir la cantidad radiación solar directa o global incidente, contempla un proceso completamente estocástico.
Para superar este problema, muchos investigadores han optado por emplear métodos inteligentes (Grimaccia et al., 2011; Kashyap et al., 2015) debido a su capacidad de representar procesos lineales y no lineales con precisión, así como de estimar un sistema del cual no se conoce claramente su dinámica interna obteniendo mejores resultados que por métodos puramente estadísticos (Pitalúa-Díaz et al., 2019; Ruz-Hernandez et al., 2019).

Un sistema inteligente es un procedimiento de aprendizaje continuo con el objetivo de generar la salida más cercana posible a la real de referencia. Entre las técnicas más conocidas se encuentra la Red Neuronal Artificial (ANN por sus siglas en inglés), lógica difusa y la Red Adaptable basada en Sistemas de Inferencia Difusa (ANFIS por sus siglas en inglés) (Boyacioglu et al., 2010; Leva et al., 2017; Matich, 2001; Wen-Tao et al., 2017) siendo este último la combinación de los dos primeros, adoptando las fortalezas de cada uno. La parte de la lógica difusa en el ANFIS transforma las ideas, tal cual el cerebro humano las pensaría, en valores medibles de 0 a 1 (Pitalúa et al., 2008) y la parte de la ANN permite el aprendizaje constante del sistema (Chen et al., 2008). Ambas partes trabajan en conjunto para reducir a su mínimo posible el error entre la estimación y los datos reales.

El objetivo de este trabajo es estimar satisfactoriamente la potencia eléctrica de un sistema fotovoltaico (SFV) de $3.1 \mathrm{~kW}$ ubicado en la ciudad de Hermosillo, Sonora usando las variables meteorológicas del sitio aplicando el ANFIS como técnica inteligente en su modalidad de predictor. La importancia de lograr exitosamente un modelo de estimación satisfactorio es crucial en estudios de precisión o en tareas de administración donde ciertas diferencias entre lo estimado y lo real pueden llevar a problemas económicos o pérdida de información.

Consecuentemente, se tendrá un mejor entendimiento de la energía solar como recurso renovable, así como de las variables meteorológicas involucradas en su generación; contribuyendo al avance científico del área al lograr un modelo SFV mexicano capaz de implementarse en cualquier ubicación. 
Se busca que esta aportación logre mejorías en la eficiencia energética y futuros estudios basados en SFV tales como de dimensionamiento local o de controlabilidad y observabilidad.

La sección Introducción expone una breve explicación de los sistemas fotovoltaicos y técnicas inteligentes más comúnmente utilizadas, así como la problemática abordada en este trabajo y su importancia. La sección Metodología explica el concepto y teoría de la técnica inteligente ANFIS; así mismo, detalla el proceso que fue llevado a cabo para la implementación del modelo predictivo inteligente.

La sección Resultados y discusión se compone de los resultados obtenidos al comparar la señal de salida del ANFIS con la real recopilada por la planta; de igual manera, se presenta un análisis del error entre ambas señales y su coeficiente de determinación para evaluar la precisión. La sección Agradecimientos presenta a las instituciones involucradas que apoyaron en este estudio. Finalmente, se presentan las secciones Conclusión y Referencias del trabajo.

\section{Metodología}

\section{ANFIS}

Como bien se mencionó en la Introducción, la técnica inteligente a implementar es un ANFIS el cual combina dos técnicas inteligentes, red neuronal y lógica difusa, para ganar sus fortalezas y superar las debilidades de cada una. La Figura 1 muestra la estructura clásica de un ANFIS el cual consta de 2 entradas $x_{1}$ y $x_{2}$, cada una con dos funciones de membresía las cuales convierten los valores de entrada a valores entre 0 y 1 para generar las sentencias o reglas difusas propias de la lógica difusa; estas son simbolizadas por $\mathrm{A}_{1}, \mathrm{~A}_{2}, \mathrm{~B}_{1}$ y $\mathrm{B}_{2}$ en la Capa 1 y también se le conoce como la capa de la "fuzificación". La Capa 2 consta de nodos redondos u operaciones fijas. La ecuación 1 representa la salida obtenida entre cada dos funciones de membresía donde $\mu$ es la función de membresía, $A_{i}$ es la etiqueta lingúistica y $w_{i}$ es la salida o fuerza disparo de cada regla difusa o peso (Pitalúa et al., 2008).

$$
w_{i}=\mu_{A_{i}}\left(x_{1}\right) \times \mu_{B_{i}}\left(x_{2}\right), \quad i=1,2 .
$$

La Capa 3 consta de la normalización de dichas fuerzas. La Capa 4 involucra ciertos parámetros conocidos como "consecuentes" los cuales permiten la "defuzificación” para generar resultados en el rango de la salida original. Finalmente, la Capa 5 concluye con un promedio de todas las salidas de la Capa 4 para encontrar un único resultado de salida descrito por la ecuación 2 , donde $f_{i}$ corresponde a la unión de los valores de entrada con los parámetros consecuentes de la Capa 4.

$$
\text { Salida total }=\frac{\sum_{i} w_{i} f_{i}}{\sum_{i} w_{i}}
$$

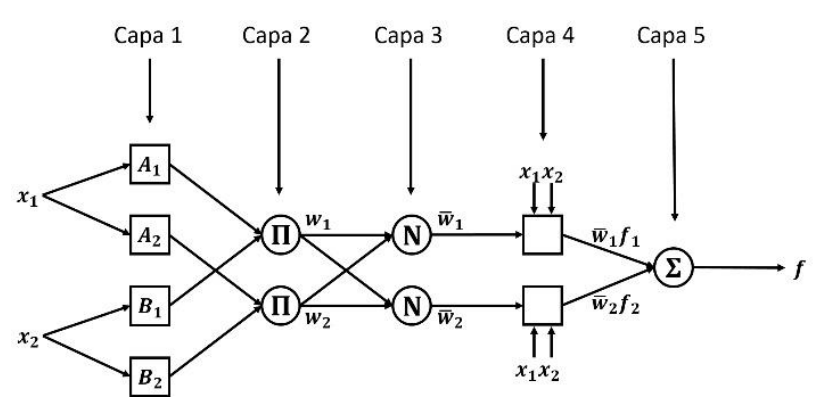

Figura 1 Estructura de un ANFIS

La parte de la red neuronal permite que los pesos y parámetros involucrados se ajusten continuamente a través del proceso de aprendizaje. El aprendizaje o entrenamiento involucrado para este trabajo se conoce como híbrido ya que contempla mínimos cuadrados para estimar el resultado de una capa hacia la siguiente, y la descendencia del gradiente que propaga el error de una capa hacia la anterior para minimizarlo ajustando los parámetros.

Para la descendencia del gradiente la ecuación 3 muestra la llamada función de costo descrita a través de la diferencia entre el valor estimado $T_{m, p}$ y el real $O_{m, p}$, también conocido como el error $E_{p}$ entre ambas señales. La descendencia del gradiente contempla derivadas parciales con respecto a $\alpha$ (parámetro genérico del sistema) como lo demuestra la ecuación 4 y ésta a su vez se basa en la ecuación 5 la cual aplica la razón de aprendizaje representada por $\eta$ (Jang, 1993).

$$
\begin{aligned}
& E_{P}=\sum_{m=1}^{L}\left(T_{m, p}-O_{m, p}^{L}\right)^{2} \\
& \frac{\partial E_{P}}{\partial \alpha}=\sum_{p=1}^{P} \frac{\partial E_{P}}{\partial \alpha}
\end{aligned}
$$




$$
\Delta \alpha=-\eta \frac{\partial E_{P}}{\partial \alpha}
$$

\section{Modalidad y técnica de entrenamiento}

Para la obtención de un modelo ANFIS, cierta cantidad de los datos recabados se usa para lograr el entrenamiento y una vez logrado se prueba la estimación resultante con datos nuevos o frescos. Este trabajo incorpora el sistema inteligente en su modalidad de predictor el cual se describe por la ecuación 6 , donde $K$ representa la muestra considerada actualmente, $y$ es la salida, $X$ es el vector de variables de entrada y $\hat{y}$ es la salida estimada. Se puede observar que para encontrar la salida actual se requiere el vector de entrada contemplando la muestra anterior y la salida estimada dos veces anterior.

$$
\left.\begin{array}{l}
y(k)=f(X(k-1), \hat{y}(k-2)) \\
X(k-1)=x_{1}(k-1), \cdots, x_{i}(k-1)
\end{array}\right\}
$$

La Figura 2 representa gráficamente el proceso de entrenamiento de acuerdo a lo mencionado anteriormente. El ANFIS toma las variables de entrada de la planta (sistema fotovoltaico) y considera la muestra anterior, mientras que la salida de la planta también es ingresada como entrada, pero considerando la muestra dos veces anterior. El resultado estimado se compara con la salida real de la planta y el error se utiliza para el entrenamiento del ANFIS. Una vez alcanzado el aprendizaje, se modifica el esquema generando una autorecursividad (Figura 3) con la finalidad de que la entrada obtenida de la salida de la planta sea recolectada por la misma salida estimada del sistema ANFIS, de esta manera el modelo inteligente sólo depende de las variables meteorológicas como variables externas.

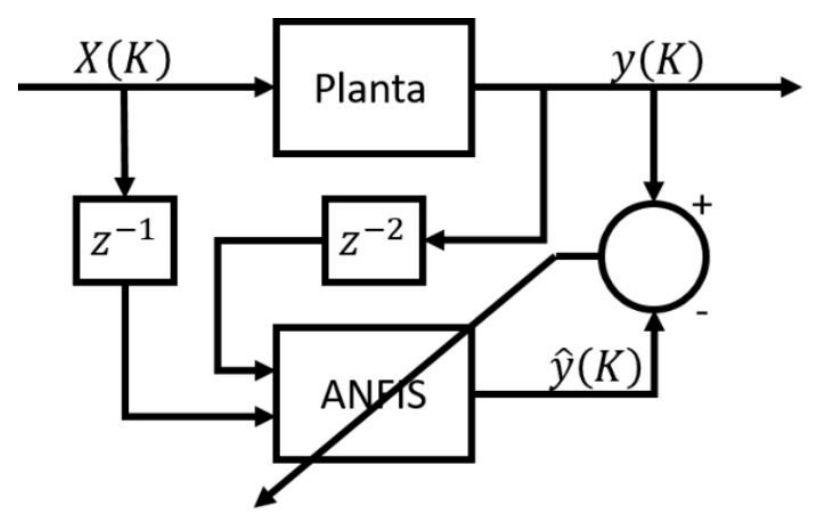

Figura 2 Esquema de entrenamiento

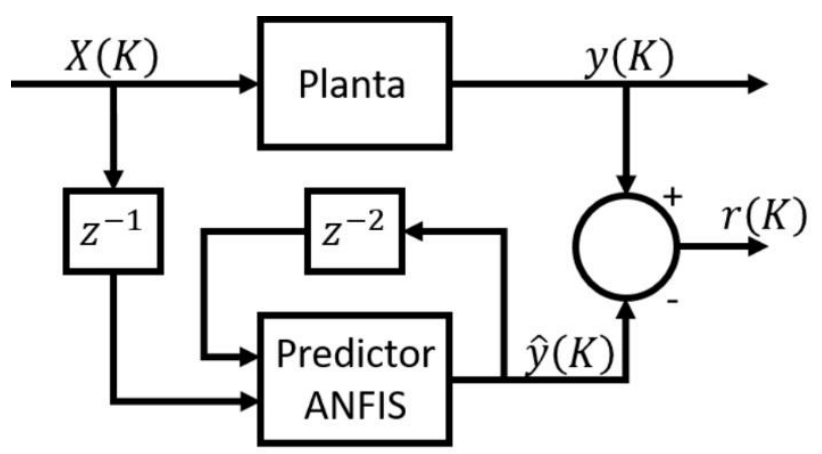

Figura 3 Esquema como predictor

Las variables utilizadas para el aprendizaje fueron: radiación solar con 4 funciones de membresía (FM) de tipo triangular, temperatura exterior con $3 \mathrm{FM}$ de tipo gaussiana, humedad con 3 FM de tipo trapezoidal, presión atmosférica con $3 \mathrm{FM}$ de tipo triangular, velocidad del viento con 3 FM de tipo triangular, dirección del viento con $3 \mathrm{FM}$ de tipo triangular, hora del día con 3 FM de tipo triangular y la potencia con 4 FM de tipo gaussiana. Cada variable fue registrada cada 5 minutos durante 6 meses obteniéndose aproximadamente 16,300 datos por variable. La Tabla 1 muestra la simbología utilizada para representar cada variable.

\begin{tabular}{|l|r|}
\hline \multicolumn{1}{|c|}{ Variable } & Simbología \\
\hline Radiación solar & $x_{1}$ \\
\hline Dirección del viento & $x_{2}$ \\
\hline Humedad & $x_{3}$ \\
\hline Presión atmosférica & $x_{4}$ \\
\hline Temperatura & $x_{5}$ \\
\hline Velocidad del viento & $x_{6}$ \\
\hline Hora del día & $x_{7}$ \\
\hline Potencia eléctrica & $y$ \\
\hline
\end{tabular}

Tabla 1 Simbología de las variables del modelo

Finalmente, una vez que el sistema ha sido debidamente entrenado se implementa la recursión de la potencia estimada para la modalidad de predictor, un acotamiento de la señal y condiciones iniciales.

\section{Consideraciones generales}

Teniendo en cuenta que el entrenamiento necesita de " $n$ " iteraciones para aprender, se concluye que el procesador que realizará el entrenamiento debe soportar la cantidad de variables de entrada, la cantidad de datos por variable, la cantidad de funciones de membresía para cada variable y la cantidad de repeticiones que tendrá el ANFIS durante su aprendizaje. 
Esto plantea un problema en el tiempo de cómputo necesario para entrenar debido a la cantidad de memoria RAM que el procesador requiere. De acuerdo a lo anterior, se optó por una arquitectura donde las variables de entrada se reparten en 3 primeros ANFIS para su entrenamiento, mientras que un cuarto ANFIS reúne los resultados y entrena con ellos para obtener una sola salida; permitiendo mejorar la estimación final y reducir considerablemente el tiempo de procesamiento. Cada una de las 3 entradas del ANFIS 4 tienen 3 FM de tipo gaussiana. La ecuación 7 describe las funciones implementadas para entrenar cada ANFIS siendo $s_{i}$ su respectivo resultado.

$$
\left.\begin{array}{c}
s_{1}(K)=f\left(x_{1}(K-1), x_{2}(K-1), x_{3}(K-1), y(K)\right) \\
s_{2}(K)=f\left(x_{4}(K-1), x_{5}(K-1), y(K)\right) \\
s_{3}(K)=f\left(x_{6}(K-1), x_{7}(K-1), y(K-2), y(K)\right) \\
s_{4}(K)=f\left(s_{1}(K), s_{2}(K), s_{3}(K), y(K)\right)
\end{array}\right\}
$$

Debido a que la potencia máxima que puede generar el sistema fotovoltaico real es de $3.1 \mathrm{~kW}$ y la mínima es de cero, se aplica un acotamiento entre dichos valores para así evitar posibles picos de potencia imposibles físicamente. Las condiciones iniciales son requeridas ya que la potencia como entrada está considerada con un retardo $(K-2)$ respecto a las entradas restantes $(K-1)$; por lo que, para las dos primeras estimaciones a causa del retardo de segundo orden, el valor de potencia será considerado como el de la planta y consecuentemente se toma en cuenta el generado por la estimación gracias a la recursividad.

\section{Resultados y discusión}

La Figura 4 muestra la estimación del modelo ANFIS implementado, considerando los datos del entrenamiento, en la cual se aprecia un resultado aceptable; sin embargo, para probar que dicho modelo logra un buen desempeño durante un período de tiempo fuera del de aprendizaje, se utilizaron 3 meses de datos recientes. La cantidad de reglas difusas para cada ANFIS fue de 36, 9, 36 y 27, respectivamente.

La figura 5 muestra un resultado satisfactorio en el cual el comportamiento del sistema neuro-difuso es muy cercano al real, incluso para los días donde la potencia fotovoltaica no fue la ideal, teniendo crestas por debajo del valor promedio y comportamientos estocásticos.

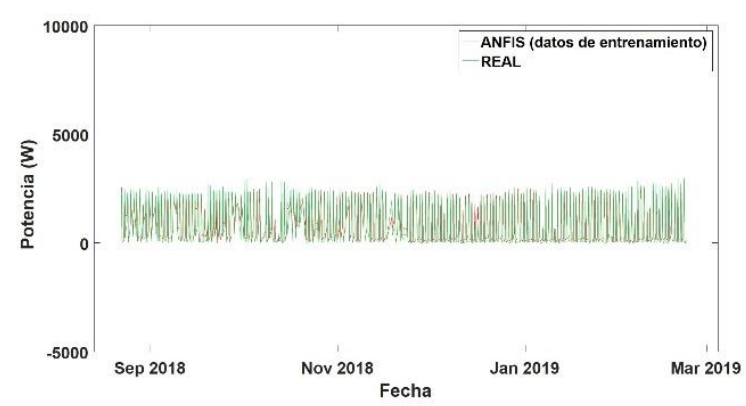

Figura 4 Comparación entre la potencia real y la estimación del ANFIS para los datos de entrenamiento

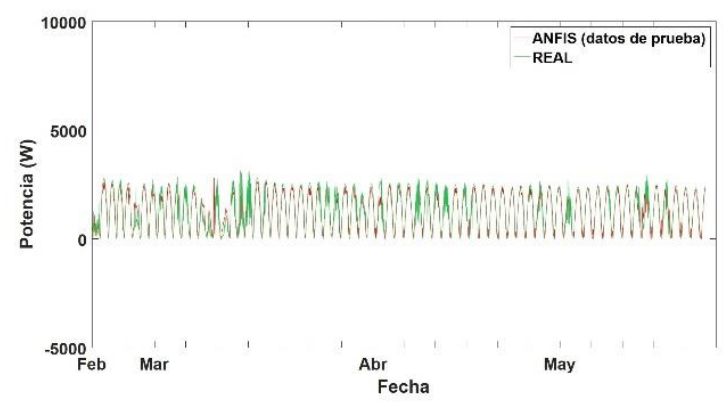

Figura 5 Comparación entre la potencia real y la estimación del ANFIS para los datos frescos

Para poder determinar el grado de satisfacción de los resultados obtenidos fue necesario aplicar un método que indique la cercanía de la estimación obtenida con respecto al comportamiento real. Para esto se empleó el coeficiente de determinación y el análisis del error entre ambas señales utilizando el software MATLAB.

El coeficiente de determinación, basado en el coeficiente de Pearson, está definido como el porcentaje de la variación de los valores de la variable dependiente que pueden ser explicados como cambios en la variable independiente. En otras palabras, un coeficiente de determinación $R^{2}=0.23$ simboliza que el $23 \%$ de la variable dependiente está ligado a los cambios de la variable independiente (Taylor, 1990). El análisis del error fue realizado aplicando el MAE (Mean Absolute Error o Error Medio Absoluto) y el MAPE (Mean Absolute Percentage Error o Error Porcentual Medio Absoluto) como técnicas de evaluación de precisión por ser de los más utilizados en sistemas inteligentes. MAE y MAPE están descritas por las ecuaciones 8 y 9 , respectivamente, donde $N$ es la cantidad total de muestras, $s$ es la muestra a considerar, $P_{m}$ es el valor de potencia real o medida y $P_{e}$ es la potencia estimada por el ANFIS (Dev et al., 2018; Pitalúa-Díaz et al., 2019; Ruz-Hernandez et al., 2019). 
A partir de la Figura 6 y Figura claramente se logra apreciar un comportamiento lineal de los datos puntuales (verde) el cual se comprueba con la línea de tendencia (roja) indicando que la estimación del modelo implementado es lineal y directamente proporcional a los valores reales. A su vez, la Tabla 2 detalla los valores obtenidos por cada método evaluado. Es posible observar que los resultados del error mostraron valores bajos estando alrededor del 7\% entre ambas señales; y el coeficiente de determinación demostró el buen desempeño del modelo inteligente alcanzando un valor de 0.9742 para los datos frescos, muy cercano al 1.

$$
\begin{aligned}
& M A E=\frac{\sum_{s=1}^{N}\left|P_{m}-P_{e}\right|}{N} \\
& M A P E_{\%}=\frac{\sum_{s=1}^{N}\left|\frac{P_{m}-P_{e}}{\max \left(P_{m}\right)-\min \left(P_{m}\right)}\right|}{N}
\end{aligned}
$$

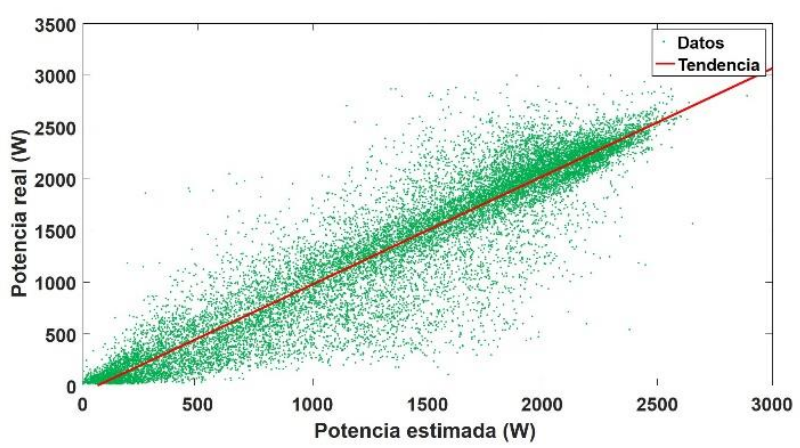

Figura 6 Gráfica de dispersión entre la potencia estimada y la potencia real (datos de entrenamiento)

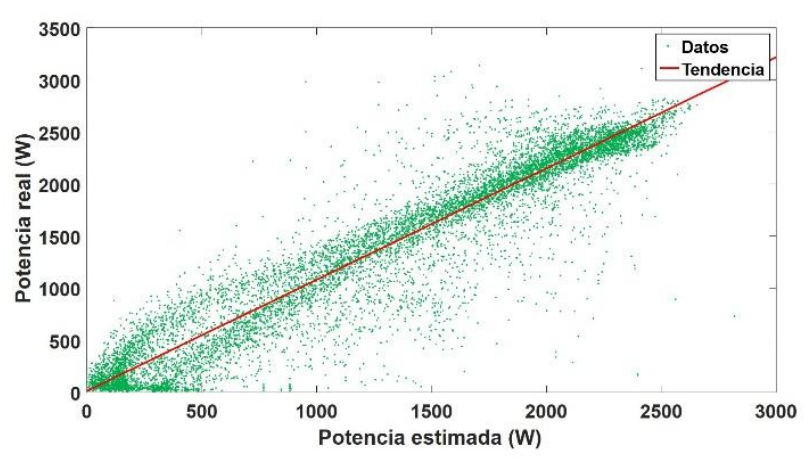

Figura 7 Gráfica de dispersión entre la potencia estimada y la potencia real (datos frescos)

\begin{tabular}{|l|l|l|}
\hline Método & \multicolumn{1}{l}{ Entrenamiento } & \multicolumn{1}{c|}{ Prueba } \\
\hline $\mathrm{R}^{2}$ & 0.9662 & 0.9742 \\
\hline MAE & $207.3163 \mathrm{~W}$ & $218.6996 \mathrm{~W}$ \\
\hline MAPE & $6.9211 \%$ & $6.9851 \%$ \\
\hline
\end{tabular}

Tabla 2 Resultados de valores de precisión

\section{Agradecimientos}

Se agradece a la Universidad Autónoma del Carmen (UNACAR) y a la Universidad de Sonora (UNISON) por el apoyo para el desarrollo de este trabajo. De igual manera, se reconoce al Consejo Nacional de Ciencia y Tecnología (CONACYT) por su apoyo con el programa de beca para maestría y la beca de movilidad 291249.

\section{Conclusión}

Este trabajo demostró que la técnica inteligente ANFIS logra una estimación exitosa de la potencia eléctrica en un SFV al reproducir satisfactoriamente su comportamiento contemplando las variables meteorológicas del sitio, alcanzando un $97.42 \%$ para el coeficiente de determinación indicando una alta relación entre la estimación y la lectura real. Así mismo, los errores implementados describieron valores pequeños tanto en unidades de potencia como en porcentaje siendo de $218.6996 \mathrm{~W}$ y $6.9851 \%$, respectivamente, reforzando la utilidad y ventaja de usar el ANFIS como técnica inteligente.

\section{Referencias}

ADRASE. (2018). Acceso a Datos de Radiación Solar en España. Disponible en http://www.adrase.com/

Awan, A., Zubair, M., P., P., Abokhalil, A., Awan, A. B., Zubair, M., ... Abokhalil, A. G. (2018). Solar Energy Resource Analysis and Evaluation of Photovoltaic System Performance in Various Regions of Saudi Arabia. Sustainability, $\quad 10(4), \quad 1129$. https://doi.org/10.3390/su10041129

Boyacioglu, M. A., \& Avci, D. (2010). An Adaptive Network-Based Fuzzy Inference System (ANFIS) for the prediction of stock market return: The case of the Istanbul Stock Exchange. Expert Systems with Applications, $37(12)$,

7908-7912. https://doi.org/10.1016/J.ESWA.2010.04.045

Chen, S. H., Jakeman, A. J., \& Norton, J. P. (2008). Artificial Intelligence techniques: An introduction to their use for modelling environmental systems. Mathematics and Computers in Simulation, 78(2-3), 379-400. https://doi.org/10.1016/J.MATCOM.2008.01.0 28

ARELLANO-VALMAÑA, Fernando, RUZ-HERNANDEZ, Jose Antonio y PITALÚA-DÍAZ, Nun. Modelo predictivo inteligente para la generación de energía eléctrica en un sistema fotovoltaico. Revista de Ingeniería Eléctrica. 2019 
Dev, S., AlSkaif, T., Hossari, M., Godina, R., Louwen, A., \& van Sark, W. (2018). Solar Irradiance Forecasting Using Triple Exponential Smoothing. In 2018 International Conference on Smart Energy Systems and Technologies (SEST) (pp.

1-6).

IEEE.

https://doi.org/10.1109/SEST.2018.8495816

Dudley, B. (2018). BP energy outlook 2040. BP Plc2. Disponible en https://www.bp.com/content/dam/bp/businesssites/en/global/corporate/pdfs/energyeconomics/energy-outlook/bp-energy-outlook2018.pdf

Elshurafa, A. M., Albardi, S. R., Bigerna, S., \& Bollino, C. A. (2018). Estimating the learning curve of solar PV balance-of-system for over 20 countries: Implications and policy recommendations. Journal of Cleaner Production, 196, 122-134. https://doi.org/10.1016/J.JCLEPRO.2018.06.01 6

Grimaccia, F., Mussetta, M., \& Zich, R. (2011). Neuro-fuzzy predictive model for PV energy production based on weather forecast. In 2011 IEEE International Conference on Fuzzy Systems (FUZZ-IEEE 2011) (pp. 2454-2457). IEEE.

https://doi.org/10.1109/FUZZY.2011.6007687

Jang, J.-S. R. (1993). ANFIS: adaptive-networkbased fuzzy inference system. IEEE Transactions on Systems, Man, and Cybernetics, 23(3), 665-685. https://doi.org/10.1109/21.256541

Järvelä, M., Valkealahti, S., Järvelä, M., \& Valkealahti, S. (2017). Ideal Operation of a Photovoltaic Power Plant Equipped with an Energy Storage System on Electricity Market. Applied $\quad$ Sciences, 7(7), 749. https://doi.org/10.3390/app7070749

Kashyap, Y., Bansal, A., \& Sao, A. K. (2015). Solar radiation forecasting with multiple parameters neural networks. Renewable and Sustainable Energy Reviews, 49, 825-835. https://doi.org/10.1016/J.RSER.2015.04.077
Kazem, H. A., Yousif, J. H., \& Chaichan, M. T. (2016). Modeling of daily solar energy system prediction using support vector machine for Oman. International Journal of Applied Engineering Research, 11(20), 10166-10172. Disponible en https://www.researchgate.net/profile/Miqdam_ Chaichan/publication/309618489_Modelling_o f_Daily_Solar_Energy_System_Prediction_usin g_Support_Vector_Machine_for_Oman/links/5 81a227908aeffb294131386/Modelling-of-

Daily-Solar-Energy-System-Prediction-usingSuppo

Leva, S., Dolara, A., Grimaccia, F., Mussetta, M., \& Ogliari, E. (2017). Analysis and validation of 24 hours ahead neural network forecasting of photovoltaic output power. Mathematics and Computers in Simulation, 131, 88-100. https://doi.org/10.1016/J.MATCOM.2015.05.0 10

Matich, D. J. (2001). Redes Neuronales: Conceptos básicos y aplicaciones. Universidad Tecnológica Nacional de México. Disponible en ftp://decsai.ugr.es/pub/usuarios/castro/MaterialRedes-Neuronales/Libros/matichredesneuronales.pdf

Perea-Moreno, A.-J., Hernandez-Escobedo, Q., Garrido, J., Verdugo-Diaz, J., Perea-Moreno, A.-J., Hernandez-Escobedo, Q., ... VerdugoDiaz, J. D. (2018). Stand-Alone Photovoltaic System Assessment in Warmer Urban Areas in Mexico. Energies, 11(2), 284. https://doi.org/10.3390/en11020284

Philibert, C. (2011). Renewable Energy Technology, Solar Energy Perspectives. Organisation for Economic Co-operation and Development/International Energy Agency (OECD/IEA), Paris. ISBN: 9264124578.

Pitalúa-Díaz, N., Arellano-Valmaña, F., RuzHernandez, J. A., Matsumoto, Y., Alazki, H., Herrera-López, E. J., ... Velázquez-Contreras, E. F. (2019). An ANFIS-Based Modeling Comparison Study for Photovoltaic Power at Different Geographical Places in Mexico. Energies, $12(14), 2662$. https://doi.org/10.3390/en12142662 
Pitalúa, N., Penaloza, U. C., Ruz-Hernandez, J. A., \& Jimenez, R. L. (2008). Introducción a los sistemas inteligentes. Departamento de Editorial Universitaria (UABC), Mexicali, Baja California, Mexico. ISBN: 978-607-7753-47-6.

Rasero, C. M. (2011). Energía solar fotovoltaica. Energía Solar Fotovoltaica, Situación Actual. Disponible en https://static.eoi.es/savia/documents/component e75553.pdf

Ruz-Hernandez, J. A., Matsumoto, Y., ArellanoValmaña, F., Pitalúa-Díaz, N., CabanillasLópez, R. E., Abril-García, J. H., ... VelázquezContreras, E. F. (2019). Meteorological Variables' Influence on Electric Power Generation for Photovoltaic Systems Located at Different Geographical Zones in Mexico. Applied Sciences, 9(8), 1649. https://doi.org/10.3390/app9081649

Taylor, R. (1990). Interpretation of the Correlation Coefficient: A Basic Review. Journal of Diagnostic Medical Sonography, 6(1), 35-39. https://doi.org/10.1177/875647939000600106

Wen-Tao, Z., Shuai, W., \& Xin-Hui, D. (2017). Research of power prediction about photovoltaic power system: Based on BP neural network. Journal of Environmental Protection and Ecology, 18(4), 1614-1623. Disponible en https://www.researchgate.net/profile/Claudia_ Moisa/publication/323144140_SUSTAINABL E_DEVELOPMENT_THROUGH_CONVERS ION_TO_ORGANIC_AGRICULTURE__IMPLICATIONS_ON_THE_FINANCIAL_I NDICATORS_OF_FIRMS/links/5c25f7bfa6fd ccfc706d4a64/SUSTAINABLEDEVELOPMENT-THROUGH-C

Zahedi, A. (2006). Solar photovoltaic (PV) energy; latest developments in the building integrated and hybrid PV systems. Renewable Energy, 31(5), 711-718. https://doi.org/10.1016/J.RENENE.2005.08.007 